\title{
Evidence for metabolism of o-xylene by simultaneous ring and methyl group oxidation in a new soil isolate
}

\author{
S. R. Bickerdike, ${ }^{1}$ R. A. Holt ${ }^{2}$ and G. M. Stephens ${ }^{1}$ \\ Author for correspondence: G. M. Stephens. Tel: +44161200 4377. Fax: +441612004399. \\ e-mail: gmstephens@umist.ac.uk
}

\footnotetext{
1 Department of Chemical Engineering, UMIST, PO Box 88, Manchester M60 1QD, UK

2 Zeneca LifeScience Molecules, PO Box 2, Billingham, Cleveland TS23 1LB, UK
}

\begin{abstract}
An o-xylene-utilizing Rhodococcus, strain B3, was isolated from enrichments with o-xylene. The pathway for 0 -xylene degradation was investigated by simultaneous adaptation experiments, studies of product formation by a mutant and fortuitous oxidation studies using trimethylbenzene isomers as substrates. Two pathways were found to operate simultaneously and both were inducible. The first pathway involved the oxidation of a methyl group to form 2-methylbenzyl alcohol, followed by oxidation via the corresponding acid to 3-methylcatechol. The second pathway involved oxidation of the aromatic ring to form a dimethylcatechol. The bulk of the evidence suggests that the initial reaction was catalysed by a monooxygenase rather than a dioxygenase, and that 2,3-dimethylphenol was produced as an intermediate.
\end{abstract}

Keywords: o-xylene metabolism, dimethylcatechols, dimethylphenols, cis-glycols

\section{INTRODUCTION}

The metabolism of $m$-xylene and $p$-xylene is well understood (Gibson \& Subramanian, 1984) but there is still some doubt about the pathway for the metabolism of the third dimethylbenzene isomer, o-xylene. A Corynebacterium sp. and a Nocardia sp. are thought to metabolize this compound by an initial dioxygenasecatalysed reaction to form a cis-glycol (Gibson \& Subramanian, 1984; Schraa et al., 1987; Fig. 1), although direct evidence for the presence of the enzyme has not yet been obtained. The same pathway probably operates in Pseudomonas stutzeri, but there is some evidence that the initial reaction may be catalysed by a monooxygenase to form a dimethylphenol instead (Baggi et al., 1987). There has hitherto been no evidence that $o$-xylene is metabolized by methyl group oxidation to form 2methylbenzyl alcohol (Gibson \& Subramanian, 1984; Baggi et al., 1987; Schraa et al., 1987), even though the other dimethylbenzene isomers are metabolized in this way (Gibson \& Subramanian, 1984).

We isolated a Rhodococcus strain which grows on $o$-xylene. By using a combination of simultaneous adaptation experiments, fortuitous oxidation studies and studies of product formation by a mutant, we obtained evidence that pathways for both ring and methyl group oxidation can operate in the same micro-organism. In addition, we present evidence that the ring oxidation pathways can be initiated by a monooxygenasecatalysed reaction.

\section{METHODS}

Isolation and growth of strain B3. Strain B3 (NCIMB 13447), identified as a Rhodococcus species (T. Dando, NCIMB, Aberdeen, personal communication), was isolated from garden soil. The isolation procedure, growth conditions and apparatus were essentially as described by Stephens \& Dalton (1986) except that MS medium pH 6.8 (Stephens \& Dalton, 1987) was used, with $o$-xylene $(0 \cdot 1 \% \mathrm{v} / \mathrm{v})$ as the carbon and energy source. When required, other carbon and energy sources were substituted for $o$-xylene. Water-insoluble organic liquids and solids were considered to be sterile and were added directly to the concentrations stated in the text; glucose and sodium acetate were added from sterile concentrated stock solutions to a final concentration of $5 \mathrm{~g} \mathrm{l}^{-1}$. Cultures grown on agar plates were provided with $o$-xylene vapour by incubation in plastic freezer boxes (approximately 11 ) containing $o$-xylene $(5 \mathrm{ml})$ in a universal bottle. Strain B3 was maintained on nutrient agar plates which were subcultured every 4 weeks and was also stored in nutrient broth at $-20^{\circ} \mathrm{C}$. All cultures were grown at $30^{\circ} \mathrm{C}$.

Construction and characterization of mutants. Strain B3 was grown on glucose to an $\mathrm{OD}_{540}$ of $0 \cdot 8$, serially diluted and plated on nutrient agar. The plates were opened in a laminarflow cabinet and exposed to a UV light positioned $15 \mathrm{~cm}$ 


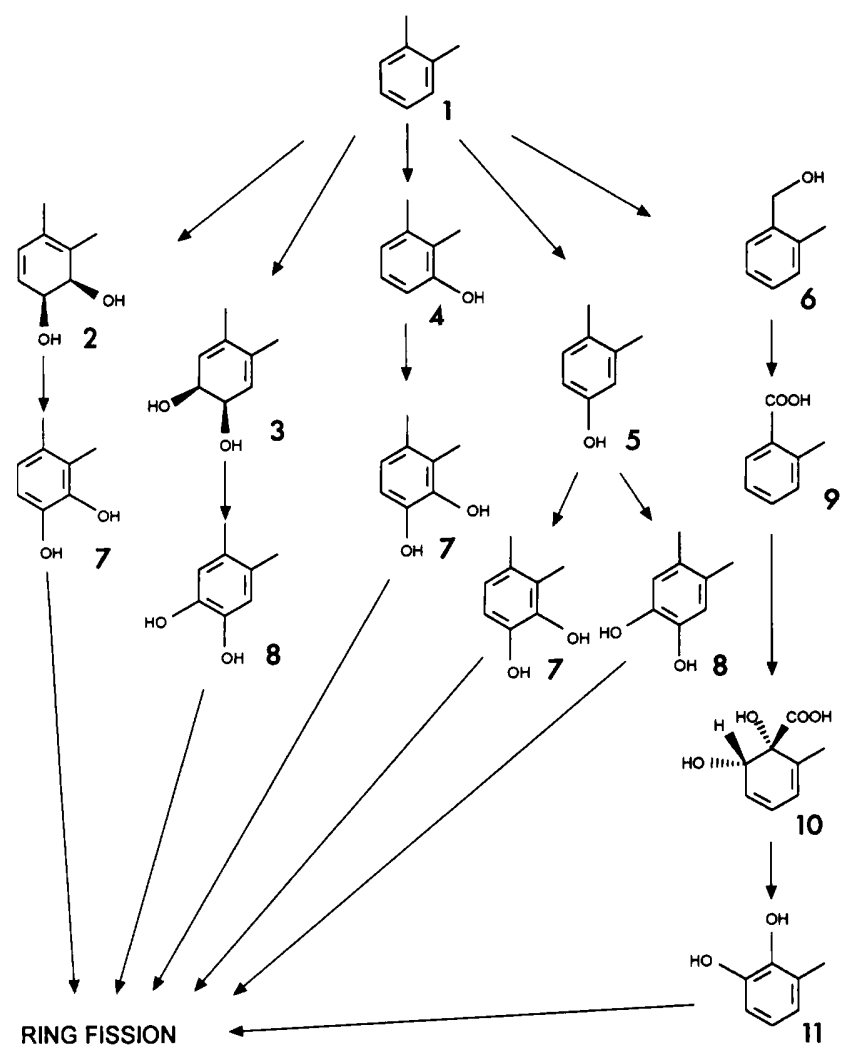

Fig. 1. Possible metabolic pathways for the metabolism of $o$-xylene. The possible routes for oxidation of o-xylene are shown as far as the corresponding catechol derivatives, which are expected to be the substrates for ring fission. For clarity, cofactors and reactants other than the pathway intermediates are not shown, nor is 2-methylbenzaldehyde. In addition, pathways involving both ring and methyl group oxidation are not shown, although it is feasible that such pathways may exist. 1, o-Xylene; 2, 1,2-dihydroxy-3,4-dimethylcyclohexa-3,5-diene; 3, 1,2-dihydroxy-4,5-dimethylcyclohexa-3,5-diene; 4, 2,3dimethylphenol; 5, 3,4-dimethylphenol; 6, 2-methylbenzyl alcohol; 7, 3,4-dimethylcatechol; 8, 4,5-dimethylcatechol; 9, 2methylbenzoic acid; 10, 1,2-dihydroxy-5-methylcyclohexa-3,5diene carboxylic acid; 11, 3-methylcatechol.

above the open Petri dish for $50 \mathrm{~s}$. The plates were then incubated at $30^{\circ} \mathrm{C}$ in the dark. Colonies were picked onto nutrient agar, MS agar containing glucose and MS agar without a carbon source. The latter cultures were incubated under $o$-xylene vapour. In this way, it was possible to identify non-auxotrophic mutants which had lost the ability to grow on $o$-xylene.

Preparation of washed cell suspensions. Strain B3 was grown on $o$-xylene or glucose to an $\mathrm{OD}_{540}$ of 0.9 . The mutant SG25/92 was grown on acetate plus 0 -xylene to an $\mathrm{OD}_{540}$ of 0.6 . The cells were harvested by centrifugation at room temperature for $10 \mathrm{~min}$ at 5000 r.p.m. in a Wiffig benchtop centrifuge and washed three times in $20 \mathrm{mM}$ potassium phosphate buffer $\mathrm{pH} 7 \cdot 0$ (KPB). The cell pellet was resuspended in $\mathrm{KPB}$ so that the $\mathrm{OD}_{540}$ was equivalent to 15 for oxygen electrode studies or 30 for all other studies.

Measurement of oxygen uptake by cell suspensions. Oxygen uptake rates were measured as described by Stephens \& Dalton (1986) except that the reaction mixture contained
$100 \mu$ cell suspension, the required volume of the substrate solution and sufficient KPB to adjust the total volume to $3 \mathrm{ml}$. Toluene $c$ is-glycol and benzene $c i s$-glycol were supplied by ICI Fine Chemicals (now Zeneca LifeScience Molecules) and were prepared as stock solutions $(150 \mathrm{mM})$ in KPB. Dimethylphenols, 2-methylbenzyl alcohol and 2methylbenzoic acid were prepared as concentrated stock solutions $(1 \mathrm{M})$ in methanol and added to the concentrations described in the text. Methanol was not metabolized and was non-toxic to strain B3. Emulsions of o-xylene and 2methylbenzaldehyde ( $150 \mathrm{mmol}^{-1}$ in $\mathrm{KPB}$ ) were prepared by vortexing for $1 \mathrm{~min}$ and were added $(100 \mu \mathrm{l}$ or $20 \mu \mathrm{l} \mathrm{re}-$ spectively) to the reaction mixture immediately.

Measurement of substrate consumption and product formation by cell suspensions. Reaction mixtures containing cell suspensions $(3 \mathrm{ml})$ and the substrate were incubated in sealed Quickfit flasks on a gyratory shaker at $30^{\circ} \mathrm{C}$ at 250 r.p.m. Dimethylphenols and 2-methylbenzyl alcohol were dissolved in methanol and added to a final concentration of $1 \mathrm{mM}$. o-Xylene and trimethylbenzenes were added as the undiluted liquid $\left(1 \mu \mathrm{lm}^{-1}\right)$. The reaction mixtures were incubated for $24 \mathrm{~h}$ unless stated otherwise. Samples were taken using a syringe.

Analytical procedures. Biomass concentrations were determined by diluting the samples in sterile medium and measuring the $\mathrm{OD}_{540}$. Metabolic products were identified by GC or GCMS analysis of culture supernatants. Absolute concentrations of the substrates and products could not be measured, either because authentic standards of some products were not available or because of the poor water solubility of the substrates, which were often present as a separate phase. Samples were prepared for analysis by removing the cells using a microcentrifuge. The supernatants were extracted into an equal volume of ethyl acetate by vortexing for $2 \mathrm{~min}$. The phases were separated by centrifugation and the ethyl acetate layer was removed for analysis either by GC or GCMS, as indicated in the text. GC analysis was done using a Perkin Elmer 310 gas chromatograph equipped with a flame ionization detector and a BP10 column $(25 \mathrm{~m} \times 0.33 \mathrm{~mm}, 0.5 \mu \mathrm{m}$ phase thickness, $\mathrm{SGE})$. Injection port and detector temperatures were maintained at $250^{\circ} \mathrm{C}$. A temperature programme was used, comprising isothermal chromatography at $80^{\circ} \mathrm{C}$ for $2.5 \mathrm{~min}$, followed by a linear temperature gradient of $30^{\circ} \mathrm{C} \mathrm{min}^{-1}$ to achieve a final temperature of $200^{\circ} \mathrm{C}$, which was held for $2.5 \mathrm{~min}$. Sample components were identified by comparing their retention times with those of authentic standards. GCMS analysis was done using a Hewlett-Packard 5890A series 1 gas chromatograph coupled to a VG Tritech TS250E mass spectrometer. Samples $(1 \mu \mathrm{l})$ were injected by splitless injection onto a DBI capillary column $(15 \mathrm{~m} \times 0.32 \mathrm{~mm}, 0.2 \mu \mathrm{m}$ phase thickness, J \& W Scientific). Sample components were resolved using a temperature programme comprising isothermal chromatography at $55^{\circ} \mathrm{C}$ for $2 \mathrm{~min}$, followed by a linear gradient of $20^{\circ} \mathrm{C} \mathrm{min}^{-1}$ to $200^{\circ} \mathrm{C}$, which was held for $2 \mathrm{~min}$, followed by a linear temperature gradient of $40^{\circ} \mathrm{C} \mathrm{min} \mathrm{m}^{-1}$ to produce a final temperature of $300^{\circ} \mathrm{C}$, which was maintained for a further $5 \mathrm{~min}$. Mass spectra were acquired using EI positive ion ionization at $70 \mathrm{eV}$. The source temperature was $175^{\circ} \mathrm{C}$. Mass spectra were scanned from 450 to 45 mass units over $1 \mathrm{~s}$ and the resolution was 300 . Analyses were conducted at the maximum sensitivity of the instrument. 
Compounds were identified by comparing mass spectra with authentic spectra from the National Institute of Standards (NIST) database, or by comparing the spectra and retention times with authentic standards when these were available. Mass spectrometry does not usually allow the determination of isomeric configuration and, therefore, it was often impossible to identify the isomer that had been formed when authentic standards were not available.

The data presented are from representative experiments, but all experiments were repeated at least twice to ensure that the results were reproducible.

\section{RESULTS}

\section{Growth on aromatic hydrocarbons}

Strain B3 could grow on o-xylene, toluene and benzene, reaching $\mathrm{OD}_{540}$ values of $0.45,0.72$ and 0.15 , respectively, when these substrates were provided at a concentration of $0.02 \%$. The high $\mathrm{OD}_{540}$ observed with toluene was probably due to the accumulation of a brown substance during growth. Cultures grown on $o$-xylene accumulated an unidentified green compound, with a maximum UV-visible absorbance at $340 \mathrm{~nm}$. We were unable to identify this compound by GCMS analysis since it could not be extracted into organic solvents. It was possible that the compound was a 2hydroxymuconic semialdehyde derivative, but attempts to identify it by UV-visible spectroscopy (Bayly et al., 1966) and conversion to the picolinic acid derivative (Dagley et al., 1960) were also unsuccessful. The only other product that could be detected was 2methylbenzoic acid, which was identified by GCMS analysis. This compound did not always accumulate, and was only ever produced in trace amounts.

When first isolated, strain B3 could grow on $m$-xylene and $p$-xylene when provided at $0.1 \%$, reaching $\mathrm{OD}_{540}$ values of 0.18 and 0.48 , respectively. When grown on $o$-xylene at the same concentration the $\mathrm{OD}_{540}$ was $2 \cdot 2$. However, the ability to grow on $m$-xylene or $p$-xylene was lost after the organism had been maintained in the laboratory for about 6 months. The experiments described below were done with the laboratory-adapted strain. There was never any growth on 1,2,3trimethylbenzene, 1,2,4-trimethylbenzene or 1,3,5trimethylbenzene, even when the concentration was reduced from $0 \cdot 1$ to $0 \cdot 01 \%$.

\section{Growth of strain B3 on potential intermediates of o-xylene degradation}

It was not possible to test for growth on the isomers of $o$-xylene cis-glycol and of dimethylcatechol, since these compounds were not available. The analogues, benzene cis-glycol, toluene cis-glycol, catechol and the methylcatechols, were too unstable for use as growth substrates. All of the other potential intermediates (2-methylbenzyl alcohol, 2-methylbenzaldehyde, 2methylbenzoic acid, 2,3-dimethylphenol and 3,4-
Table 1. Oxidation of potential intermediates of o-xylene metabolism by cells grown on o-xylene or glucose

Oxygen uptake rates were measured by adding various substrates to cell suspensions harvested from cultures of strain B3 grown on either $o$-xylene or glucose. Substrates were added to a concentration of $5 \mathrm{mmol}^{-1}$, except where indicated. Oxidation rates are expressed as a percentage of the rate obtained with $o$-xylene.

\begin{tabular}{|lcc|}
\hline Substrate & $\begin{array}{c}\text { Rate of oxygen uptake }(\%) \\
\text { by cells grown on: }\end{array}$ \\
\cline { 2 - 3 } & o-Xylene & Glucose \\
\hline o-Xylene & 100 & 39 \\
2-Methylbenzyl alcohol* & $0 \dagger$ & 27 \\
2-Methylbenzaldehyde* & 8 & 7 \\
2-Methylbenzoic acid & 22 & 8 \\
3-Methylcatechol & 60 & 25 \\
2,3-Dimethylphenol & 139 & 7 \\
3,4-Dimethylphenol & 70 & 5 \\
Toluene cis-glycol & 65 & 6 \\
Benzene cis-glycol & 56 & 6 \\
\hline
\end{tabular}

* These substrates were added to $1 \mathrm{mmol} \mathrm{l}^{-1}$ to cell suspensions which had been grown on 0 -xylene and to $5 \mathrm{mmol} \mathrm{l}^{-1}$ to cells grown on glucose.

† This substrate inhibited endogenous oxygen uptake.

dimethylphenol) were available, but did not support growth when provided at $0 \cdot 1 \%$. However, this was due to toxicity, because the intermediates completely inhibited growth on glucose. When the concentration was reduced to $0.01 \%$, there was still no growth on the intermediates, even though $o$-xylene supported growth at this concentration. The failure of 2 -methylbenzoic acid and 2,3-dimethylphenol to support growth cannot be attributed to toxicity because there was no inhibition of growth on glucose at the reduced concentration. In contrast, 2-methylbenzyl alcohol, 2-methylbenzaldehyde and 3,4-dimethylphenol were still toxic at the reduced concentration, since the $\mathrm{OD}_{540}$ after growth on glucose in the presence of any of these substrates was approximately $24 \%$ lower than after growth on glucose alone. It was not practical to reduce the concentration any further because there would have been insufficient substrate to support detectable growth.

\section{Simultaneous adaptation experiments}

$o$-Xylene was oxidized by both $o$-xylene- and glucosegrown cells, but the rate of oxidation was $2 \cdot 6$-fold higher in the o-xylene-grown cells (Table 1 ). This indicated that the pathway(s) for metabolism of this compound were inducible. Toluene cis-glycol, benzene cis-glycol and both of the dimethylphenol isomers were oxidized by $o$-xylene-grown cells. Of these compounds, 2,3- 
dimethylphenol was oxidized at the highest rate. The ability to oxidize these substrates was inducible, since glucose-grown cells exhibited much lower rates of oxygen uptake when the substrates were added.

Although 3-methylcatechol was oxidized, all of the other intermediates of the methyl group oxidation pathway were toxic to $o$-xylene-grown cells, causing inhibition of endogenous oxygen uptake when tested at $5 \mathrm{mM}$. When the concentration was reduced to $1 \mathrm{mM}$, the aldehyde and acid were oxidized slowly but 2methylbenzyl alcohol still inhibited endogenous oxygen uptake. Nevertheless, slow oxidation of this intermediate could be detected when GC analysis was used to monitor substrate consumption (results not shown). Furthermore, 2-methylbenzaldehyde and 2methylbenzoic acid accumulated transiently, although 3 -methylcatechol could not be detected. After $24 \mathrm{~h}$, all of the substrate and the accumulated products had been completely consumed.

Glucose-grown cells were less sensitive to inhibition by intermediates of the pathway for methyl group oxidation, and the cells were able to oxidize all of the intermediates, including 2-methylbenzyl alcohol, when they were provided at $5 \mathrm{mM}$. 3-Methylcatechol and 2methylbenzoic acid were oxidized more slowly than by $o$-xylene grown cells, which suggests that these activities were induced by growth on $o$-xylene. In contrast, oxygen uptake stimulated by 2-methylbenzyl alcohol was only detectable in glucose-grown cells, whilst the rate of 2methylbenzaldehyde oxidation was the same in both glucose- and $o$-xylene-grown cells.

\section{Isolation and characterization of mutants blocked in o-xylene metabolism}

Mutants of strain B3 were constructed by treating glucose-grown cells with UV light and screening for loss of the ability to grow on o-xylene. Of 4000 potential mutants screened, $32(0.8 \%)$ had lost the ability to grow on $o$-xylene. These mutants were tested for accumulation of metabolic products during growth on acetate in the presence of $o$-xylene. Acetate was chosen as the energy source, since oxygen uptake experiments had shown that $o$-xylene oxidation was not repressed in wild-type cell suspensions that had been grown on acetate in the presence of $o$-xylene (results not shown). The culture supernatants were extracted into ethyl acetate and analysed by GCMS.

Only two of the mutants (strains SG12/92 and SG25/92) consumed the $o$-xylene and accumulated metabolic products that could be detected by GC analysis. This suggested that the remaining mutants were either regulatory mutants or were blocked at the initial step in $o$-xylene metabolism. Strain SG12/92 accumulated small quantities of 2-methylbenzyl alcohol, but the wildtype also accumulated similar concentrations of this compound when grown under the same conditions (results not shown). In contrast, strain SG25/92 consumed $90 \%$ of the $o$-xylene and accumulated a variety of products during growth. The products included an unidentified compound $(59.8 \%$ of the total peak area due to products), 3-methylcatechol $(18.6 \%)$ and 2methylbenzyl alcohol $(17 \cdot 8 \%)$. Small quantities of 2,3dimethylphenol $(0.73 \%)$, 3,4-dimethylphenol $(1.94 \%)$ and 3-methylbenzaldehyde $(1.21 \%)$ were also present, together with traces of 2-methylbenzoic acid $(0.008 \%)$. The unidentified compound was identified as a dimethylcatechol isomer by GCMS analysis. The mass spectrum resembled those of $3,4-$ and 4,5dimethylcatechol (Higson \& Focht, 1992) very closely, whilst there was much less similarity to the mass spectra of 2,3-dimethylhydroquinone and 1,3-dihydroxy-3,4dimethylbenzene (NIST library spectra). It was not possible to determine which isomer of dimethylcatechol had been formed since the 3,4- and 4,5-dimethylcatechols have very similar mass spectra. Attempts to purify the dimethylcatechol for structure determination by NMR spectroscopy were unsuccessful because it was very unstable and decomposed to form a brown material after 15-20 min. Therefore, it was impossible to establish which isomer had been produced by the mutant.

The involvement of the dimethylcatechol isomer as an intermediate of $o$-xylene metabolism was confirmed by testing wild-type cells for their ability to oxidize this compound. A solution of partially purified dimethylcatechol could be prepared from ethyl acetate extracts of supernatants of cultures of SG25/92 provided that all operations were completed before the dimethylcatechol decomposed. The ethyl acetate was evaporated and the solid material was mixed with $20 \mathrm{mM}$ potassium phosphate buffer $\mathrm{pH} 7 \cdot 0$. When the buffer was removed after $30 \mathrm{~s}$, it had dissolved only the dimethylcatechol and 2-methylbenzyl alcohol, leaving the other products as a solid residue. The solution of dimethylcatechol and 2-methylbenzyl alcohol was added immediately to a suspension of wild-type cells which had been grown on $o$-xylene. GC analysis demonstrated that the dimethylcatechol was completely consumed within $30 \mathrm{~s}$ (results not shown).

There was no evidence for the accumulation of a cisglycol, although the GCMS method was suitable for detection of the analogue, toluene cis-glycol. Methylsubstituted cis-glycols are unstable and dehydrate readily in acidic conditions (Boyd et al., 1994), and it was possible that the failure to detect a cis-glycol was due to acid-catalysed dehydration, since the $\mathrm{pH}$ sometimes fell to 6.7 during growth. For this reason, product formation was re-examined by incubating non-growing cells suspensions of the mutant with $o$-xylene in a buffer at $\mathrm{pH} 7 \cdot 3$, a $\mathrm{pH}$ value at which cis-glycols should be stable (Gibson et al., 1970). The same products were formed as in the growing cultures, except that 2,3- and 3,4-dimethylphenol could not be detected (results not shown). It was still possible that a cis-glycol had been formed but could not be detected by the GCMS procedure. Therefore, a portion of the sample was acidified by adding $3 \mathrm{M} \mathrm{HCl}(10 \mu \mathrm{l}$ per ml supernatant) prior to extraction and analysis, since this would cause any cis-glycols to dehydrate to form the corresponding 
Table 2. Oxidation of trimethylbenzene isomers by strain B3

Strain B3 was grown on $o$-xylene or glucose. The cells were harvested and incubated with trimethylbenzenes for $24 \mathrm{~h}$, except for the experiments marked *, where the peaks due to dihydroxytrimethylbenzene and 3,4-dimethylbenzoic acid were too large to be resolved from each other after $24 \mathrm{~h}$ incubation, and the reaction mixture was therefore incubated for only $3 \mathrm{~h}$. The products were extracted and identified by GCMS. Where possible, isomers were identified by comparing the retention times with those of authentic standards; otherwise the position of the substituents is not specified. The peak areas (arbitrary units) for each product are indicated as a measure of their relative concentrations.

\begin{tabular}{|llcc|}
\hline Substrate & \multicolumn{1}{c}{$\begin{array}{c}\text { Product } \\
\end{array}$} & & $\begin{array}{c}\text { Peak area of product formed } \\
\text { by cells grown on: }\end{array}$ \\
\cline { 3 - 4 } & & Glucose \\
\hline \multirow{2}{*}{ 1,3,5-Trimethylbenzene } & 2,4,6-Trimethylphenol & $24 \cdot 0$ & $0 \cdot 952$ \\
& Dimethylbenzyl alcohol & 0 & $4 \cdot 02$ \\
& 3,5-Dimethylbenzoic acid & $24 \cdot 2$ & 0 \\
1,2,3-Trimethylbenzene & $2 \cdot 3$ & $1 \cdot 57$ \\
& 2,3,4-Trimethylphenol & $96 \cdot 2$ & $43 \cdot 8$ \\
& Dimethylbenzyl alcohol & $40 \cdot 1$ & $44 \cdot 4$ \\
& Dimethylbenzyl alcohol & $24 \cdot 4$ & $1 \cdot 09$ \\
& Dimethylbenzaldehyde & $12 \cdot 1$ & 0 \\
& 2,3-Dimethylbenzoic acid & $0 \cdot 456^{*}$ & 0 \\
& 2,3,6-Trimethylphenol & $0 \cdot 60^{*}$ & $16 \cdot 4$ \\
& 2,3,5-Trimethylphenol & $45 \cdot 4^{*}$ & $13 \cdot 1$ \\
& Dihydroxytrimethylbenzene & $7 \cdot 26^{*}$ & $5 \cdot 15$ \\
& Dimethylbenzyl alcohol & $4 \cdot 79^{*}$ & 0 \\
& Dimethylbenzaldehyde & $8 \cdot 25^{*}$ & 0 \\
\hline
\end{tabular}

phenols, which can be detected readily. There was no change in the product profile (results not shown), which indicated that a cis-glycol had not been formed.

\section{Metabolism of dimethylphenols by strain B3}

Strain B3 could oxidize both of the dimethylphenol isomers and both were also accumulated by the mutant. Therefore, the role of the dimethylphenol isomers was investigated in greater detail by studying formation of products from these substrates by harvested suspensions of strain B3 using GCMS. 2,3-Dimethylphenol was completely consumed by wild-type cells within $4 \mathrm{~h}$, and no metabolic products were detected (results not shown). In contrast, a metabolic product accumulated from 3,4-dimethylphenol, even though the substrate was completely consumed within $2 \mathrm{~h}$. The concentration of this product remained almost constant over a period of $20 \mathrm{~h}$, which suggested that it could not be metabolized. The mass spectrum was almost identical to that of the dimethylcatechol isomer produced by the mutant from $o$-xylene. However, GC analysis using a BP10 column demonstrated that the retention time of the dimethylcatechol produced from 3,4-dimethylphenol was $7.9 \mathrm{~min}$, compared with $7.64 \mathrm{~min}$ for the dimethylcatechol produced from $o$-xylene by the mutant. This indicated that the dimethylcatechols were different isomers.

\section{Fortuitous oxidation of trimethylbenzene isomers}

Several organisms which grow on other hydrocarbons can oxidize analogues of the natural substrate due to the presence of broad-specificity oxygenases which catalyse the initial steps in the degradative pathways (Colby $e t$ al., 1977; Boyd et al., 1987; Gibson et al., 1990; Kukor \& Olsen, 1990; Yen et al., 1991; Kok et al., 1992). The product of the oxygenase-catalysed reaction often accumulates and contains one or two additional oxygen atoms, depending on whether the reaction was catalysed by a monooxygenase or a dioxygenase, respectively. This provides a useful means of identifying the type of oxygenase involved in metabolism of the natural substrate (Leadbetter \& Foster, 1960; Gibson et al., 1968). For this reason, we decided to investigate fortuitous oxidation of analogues of $o$-xylene by strain B3. Trimethylbenzenes were suitable substrates because they did not support growth. Cultures of strain B3 were harvested after growth on $o$-xylene or glucose and washed, concentrated cell suspensions were incubated with the three different isomers of trimethylbenzene (Table 2). When o-xylene-grown cells were incubated with 1,3,5-trimethylbenzene, both ring and methyl group oxidation reactions were observed, since a mixture of 3,5-dimethylbenzoic acid and 2,4,6trimethylphenol accumulated. Glucose-grown cells produced a much lower concentration of 2,4,6trimethylphenol and produced small quantities of a 
dimethylbenzyl alcohol isomer (which must have been the 3,5-isomer) instead of 3,5-dimethylbenzoic acid. This indicated that both ring and methyl group oxidation were catalysed by inducible enzymes.

When $o$-xylene-grown cells were incubated with $1,2,3$ trimethylbenzene, little ring oxidation was observed, since only very small amounts of 2,3,4-trimethylphenol were produced. All of the other products resulted from methyl group oxidation. The major products were two isomers of dimethylbenzyl alcohol. These must have been the 2,3- and 2,6-isomers, since these are the only possible isomers which can be produced. 2,3Dimethylbenzoic acid was also formed, together with an unidentified isomer of dimethylbenzaldehyde. Glucosegrown cells produced a lower concentration of the products overall and the oxidation was much less extensive. Thus, very little of the dimethylbenzaldehyde isomer accumulated and 2,3-dimethylbenzoic acid was not produced at all, although the dimethylbenzyl alcohol isomers were still produced.

1,2,4-Trimethylbenzene was oxidized to a variety of products by $o$-xylene-grown cells. There were several products of ring hydroxylation, including 2,3,6- and 2,3,5-trimethylphenol. Although 2,3,6-trimethylphenol continued to accumulate, 2,3,5-trimethylphenol only accumulated during the first hour (results not shown), and was almost completely consumed after $3 \mathrm{~h}$. This coincided with an increase in the concentration of an unidentified dihydroxytrimethylbenzene isomer, which was the major product. This compound was identified from the mass spectrum, since standards were not available. The compound could have been either 1,2dihydroxy-3,4,6-trimethylbenzene or 1,3-dihydroxy2,4,5-trimethylbenzene, but library spectra were not available for these compounds. It was unlikely that the product was 1,4-dihydroxy-2,3,6-trimethylbenzene because the mass spectrum did not resemble the library spectrum for that isomer. No further attempts were made to identify this product because it was unstable. Various products of methyl group oxidation were also detected. It was not possible to determine which isomers of the products had been formed since standards were not available, except in the case of 3,4-dimethylbenzoic acid. Only two of the three possible isomers of dimethylbenzyl alcohol were produced, even after $24 \mathrm{~h}$ incubation (results not shown). Only one of the possible dimethylbenzaldehyde isomers accumulated. This compound, together with one of the dimethylbenzyl alcohol isomers, continued to accumulate. However, the other dimethylbenzyl alcohol isomer had been consumed after $3 \mathrm{~h}$ and this coincided with the production of 3,4dimethylbenzoic acid. The other isomers of dimethylbenzoic acid could not be detected. The acid must have been produced from the 3,4-isomer of dimethylbenzyl alcohol.

Glucose-grown cells produced much less of the dihydroxytrimethylbenzene isomer from 1,2,4trimethylbenzene, and a correspondingly higher concentration of 2,3,5-trimethylphenol accumulated. This indicated that the enzyme needed to convert the phenol to the dihydroxybenzene derivative was inducible. Both of the dimethylbenzyl alcohol isomers were still produced, but one of them was produced in smaller amounts. Furthermore, the aldehyde and acid were not produced at all. The total concentration of products after $24 \mathrm{~h}$ was much lower than the total product concentration formed after $3 \mathrm{~h}$ when $o$-xylene-grown cells were used. In conclusion, the fortuitous oxidation studies demonstrated that both ring and methyl group oxidation could occur simultaneously and that both pathways were induced during growth on o-xylene.

\section{DISCUSSION}

In the past, the pathway for $o$-xylene metabolism has been investigated by identifying products accumulating during growth, by testing for growth on the possible intermediates and by simultaneous adaptation experiments (Gibson \& Subramanian, 1984; Schraa et al., 1987; Baggi et al., 1987). In one case, the findings have been confirmed by enzyme studies (Schraa et al., 1987). We also used these techniques, with the exception of the last one. Enzyme studies were not possible because the cells were very resistant to breakage for the preparation of cell-free extracts. Most of the techniques depend upon the ability of the cells to metabolize intermediates of the three possible pathways for $o$-xylene degradation. However, there are a number of problems with the physical and chemical properties of the intermediates which can cause difficulties with the interpretation of experimental data. The first problem is that the dimethylphenols and the intermediates of the methyl group oxidation pathways are poorly soluble in water, which means that low oxidation rates may be due to lack of availability of the substrate. We solved this problem by dissolving the intermediates in methanol before adding them to reaction mixtures. For the first time, this made it possible to detect oxidation of intermediates of the pathway for methyl group oxidation by an $o$-xylene-degrading bacterium. Secondly, we found that several of the compounds were toxic to strain B3. Although Schraa et al. (1987) showed that the intermediates do not kill $o$-xylene-degrading bacteria, we demonstrated that they can inhibit growth and oxygen uptake even at very modest concentrations. This made it difficult to interpret our growth studies and simultaneous adaptation experiments. Furthermore, this finding suggests that previous observations that these intermediates are not usually oxidized or do not support growth (Baggi et al., 1987; Schraa et al., 1987) should be interpreted with caution. Because of these problems, we decided to use additional, complementary techniques to study the degradation of $o$-xylene.

The first of these techniques involved the study of blocked mutants. We were surprised to find that of the 32 blocked mutants isolated, only one accumulated metabolic products. This mutant accumulated 3methylcatechol and an unidentified dimethylcatechol, which indicated that it was defective in ring fission or in a later step in the pathway for $o$-xylene metabolism. The accumulation of two different catechol isomers indicated 
that two routes for $o$-xylene oxidation were operating. Furthermore, the mutant must either have been a double mutant or the two pathways must converge on a single ring fission sequence. Earlier intermediates of the pathways for both ring and methyl group oxidation also accumulated, presumably due to displacement of the equilibria of the initial reactions as a result of accumulation of the catechols. This yielded valuable information about the metabolic pathways for $o$-xylene metabolism.

The second technique involved studying fortuitous oxidation of trimethylbenzenes to identify the type of oxygenase involved in $o$-xylene metabolism. These substrates were chosen because they did not support growth. Furthermore, the structures and electronic configurations are very similar to those of $o$-xylene. This is important because oxygenases are known to catalyse unexpected types of reactions if there are significant departures from the structure of the natural substrate (May \& Abbott, 1972; Helmbrook \& Sligar, 1981; Dalton et al., 1993). It is even possible for dioxygenases to act as monooxygenases (Wackett et al., 1988), and vice versa (Wende et al., 1982). However, trimethylbenzenes are sufficiently similar to $o$-xylene to expect that they would be metabolized by the same type of reaction(s).

By using a combination of all these techniques, we obtained evidence for the simultaneous operation of both the ring and methyl group oxidation pathways. Although the intermediates of the methyl group oxidation pathway did not support growth because they were very toxic, we were able to detect oxidation of the intermediates when the concentrations were reduced to non-toxic levels. Furthermore, the wild-type accumulated 2-methylbenzoic acid during growth on $o$-xylene, 2-methylbenzyl alcohol when grown on $o$-xylene plus acetate, and dimethylbenzylalcohols, dimethylbenzaldehydes and dimethylbenzoic acids during oxidation of trimethylbenzenes. Finally, the mutant, SG25/92, accumulated 2-methylbenzyl alcohol, 2methylbenzaldehyde, 2-methylbenzoic acid and 3methylcatechol. This provides conclusive evidence that strain B3 can metabolize $o$-xylene by oxidation of a methyl group, at least to the level of 3-methylcatechol, the putative substrate for ring fission. This pathway was previously believed not to operate in $o$-xylene-degrading bacteria (Schraa et al., 1987; Baggi et al., 1987).

Evidence was also obtained that strain B3 oxidized $o$-xylene by direct oxidation of the aromatic ring to form an unidentified dimethylcatechol isomer. This compound accumulated when the mutant, SG25/92, was grown with $o$-xylene and it was oxidized completely and rapidly by the wild-type strain. The dimethylcatechol could have been produced as an intermediate of a pathway initiated by either a dioxygenase- or a ring monooxygenase-catalyzed reaction (Fig. 1). The dioxygenase pathway would involve oxidation of $o$-xylene to either 1,2-dihydroxy-3,4-dimethylcyclohexa-3,5-diene or 1,2-dihydroxy-4,5-dimethylcyclohexa-3,5-diene, the two possible isomers of $o$-xylene cis-glycol, followed by dehydrogenation to form the 3,4- or 4,5-isomers of dimethylcatechol, respectively. Production of the dimethylcatechol by the ring monooxygenase pathway would involve two sequential monooxygenase-catalysed reactions, with the intermediate formation of a dimethylphenol isomer.

There is little evidence for the involvement of a dioxygenase in $o$-xylene metabolism by strain B3. Although both toluene cis-glycol and benzene cis-glycol were oxidized inducibly by strain B3, 2,3-dimethylphenol was oxidized at more than twice the rate of either of the cis-glycols. Furthermore, there was no evidence for accumulation of cis-glycols from $o$-xylene by the mutant or from trimethylbenzenes by the wild-type, even when precautions were taken to eliminate or control acid-catalysed dehydration of cis-glycols.

Most of the evidence supports the operation of a monooxygenase-catalysed reaction as the initial step in the ring oxidation pathway. Firstly, the mutant accumulated both 2,3- and 3,4-dimethylphenol from $o$-xylene. Taken in isolation, this finding does not provide conclusive evidence for the monooxygenasecatalysed reaction, since it is possible that these products could also accumulate due to chemical dehydration of cis-glycols produced in a dioxygenase-catalysed reaction. However, direct evidence for the presence of a ring monooxygenase was obtained from the fortuitous oxidation studies. Thus, the wild-type was able to oxidize 1,3,5-trimethylbenzene to 2,4,6-trimethylphenol and 1,2,4-trimethylbenzene to 2,3,6-trimethylphenol. It would be impossible to produce these trimethylphenol isomers by dehydration of a cis-glycol, because the corresponding cis-glycols cannot be formed in the first place. The hydroxyl group in each of these products is flanked by two methyl groups. The position of the methyl groups is such that it would not be possible to insert hydroxyl groups at the two adjacent carbon atoms to form the cis-glycols by a dioxygenase-catalysed reaction. This means that the trimethylphenols could only have been formed by direct insertion of a single hydroxyl group. This provides conclusive evidence that methyl-substituted phenols can be formed directly by a monooxygenase-catalysed reaction in strain B3. The presence of such an enzyme in $o$-xylene-grown cells and the accumulation of dimethylphenols by the mutant suggests strongly that a monooxygenase, rather than a dioxygenase, is involved in $o$-xylene metabolism.

It is most likely that 2,3-dimethylphenol was the main product of monooxygenase-catalysed oxidation of $o$-xylene, rather than the 3,4-isomer. Although 2,3dimethylphenol did not support growth, this compound was oxidized completely and rapidly by strain B3, and the activity was inducible. In contrast, 3,4,dimethylphenol was oxidized at only $50 \%$ of the rate of the 2,3-isomer. Furthermore, wild-type cells could not oxidize this compound completely, with the result that a non-metabolizable dimethylcatechol isomer accumulated. This demonstrates that 3,4-dimethylphenol is 
not an intermediate of $o$-xylene metabolism. Although the mutant produced small quantities of both dimethylphenol isomers, it is probable that the 3,4isomer was formed by a side reaction during the initial oxygenase-catalysed reaction. There are precedents for such side reactions in monooxygenase-catalysed reactions, since a number of micro-organisms can hydroxylate aliphatic and aromatic hydrocarbons at more than one carbon atom, even though only one of the hydroxylated products is metabolized fully (Fredricks, 1967; Klein \& Henning, 1969; Grossebuter et al., 1979; Stephens \& Dalton, 1986; Shields et al., 1989).

In conclusion, the bulk of the evidence suggests that the ring oxidation pathway involves oxidation of $o$-xylene to 2,3-dimethylphenol rather than a cis-glycol. Further work is needed to demonstrate directly that the cells contain the o-xylene 3-monooxygenase and 2,3dimethylphenol monooxygenase activities which are necessary for dimethylcatechol formation.

It appears that both the ring and methyl group oxidation pathways are involved simultaneously in $o$-xylene metabolism. The mutant oxidized o-xylene to intermediates of both pathways, and the products of methyl group oxidation accounted for $38 \%$ of the total products. Similarly, wild-type cells oxidized all three trimethylbenzene isomers to products resulting from both ring and methyl group oxidation, and the products of methyl group oxidation represented $30 \cdot 4 \%, 50 \cdot 2 \%$ and $98.7 \%$ of the total products formed from the $1,2,4-$, 1,3,5- and 1,2,3-trimethylbenzenes, respectively. This suggests that the activities of the enzymes required for methyl group oxidation must have been comparable to those required for ring oxidation. The simultaneous adaptation experiments seem to contradict this conclusion, since the intermediates of the methyl group oxidation pathway were apparently oxidized much more slowly than those of the ring oxidation pathway. However, the intermediates were very toxic and it is possible that the experiments gave an underestimate of the activities of the enzymes involved in methyl group oxidation, due to substrate inhibition.

The fortuitous oxidation studies indicate that both the ring and the methyl group oxidation pathways were induced during growth on $o$-xylene, which lends further support to the hypothesis that both the ring and the methyl group oxidation pathways play an important part in $o$-xylene metabolism. Thus, $o$-xylene-grown, wild-type cells produced higher concentrations of products from both ring and methyl group oxidation of trimethylbenzenes than glucose-grown cells. In addition, $o$-xylene-grown cells could oxidize the trimethylbenzenes much more extensively than glucose-grown cells, producing dimethylbenzoates. The glucose-grown cells could only oxidize the substrates to the level of dimethylbenzyl alcohols, except in one case where traces of a dimethylbenzaldehyde isomer were formed in addition. The simultaneous adaptation experiments confirmed that the ring oxidation pathway was inducible. However, some of the results with intermediates of the methyl group oxidation pathway were contradictory. Although 3-methylcatechol and 2-methylbenzoic acid were oxidized inducibly, glucose-grown cells oxidized 2-methylbenzyl alcohol more rapidly than $o$-xylene-grown cells, whilst the ability to oxidize 2-methylbenzaldehyde was apparently constitutive. The anomalous results with the latter substrates may be a result of substrate toxicity. We have already suggested that the oxidation rates with o-xylene-grown cells were underestimated due to substrate inhibition. This inhibition was much less severe in glucose-grown cells, which were able to tolerate substrate concentrations that completely inhibited endogenous oxygen uptake in $o$-xylene-grown cells. This differential toxicity may explain why some intermediates were, apparently, oxidized more slowly by $o$-xylene-grown cells than by glucose-grown cells. Further work is needed to confirm this.

The hypothesis that both the ring and the methyl group oxidation pathways play an important part in o-xylene metabolism is strengthened by the observation that it was difficult to isolate mutants which accumulated intermediates of $o$-xylene metabolism. The most likely explanation for the rarity of the mutants is that only double mutations affecting both pathways or mutations affecting reactions common to both pathways were detectable by screening for loss of ability to grow on $o$-xylene. Indeed, the only product-forming mutant which was isolated accumulated both 3-methylcatechol and the unidentified dimethylcatechol isomer. This indicates that both the ring and the methyl group oxidation pathways were blocked.

Contrary to a previous suggestion (Galli et al., 1992), this study shows that the methyl group and ring oxidation pathways for $o$-xylene metabolism are, in fact, compatible and can operate within the same microorganism. Functional duplication of pathways for degradation of aromatic substrates is fairly common amongst bacteria which degrade methylbenzene derivatives (Williams \& Murray, 1974; Jenkins et al., 1987; O’Donnell \& Williams, 1991; Mahajan et al., $1994)$, so it is not entirely unexpected that $o$-xylene is also degraded using more than one pathway. However, further research is needed to elucidate the reasons for operation of multiple pathways in $o$-xylene degradation.

\section{ACKNOWLEDGEMENTS}

S.R.B. is grateful to the SERC Biotechnology Directorate and ICI Biological Products (now Zeneca LifeScience Molecules) for financial support.The GCMS and HPLC instruments were purchased using a grant from the Process Engineering Committee of the SERC (GR/F85765). We thank Paul Williams for doing the GCMS analysis and Terry Dando (NCIMB, Aberdeen) for identification of strain B3.

\section{REFERENCES}

Baggi, G., Barbieri, P., Galli, E. \& Tollari, S. (1987). Isolation of a Pseudomonas stutzeri strain that degrades o-xylene. Appl Environ Microbiol 54, 2129-2132. 
Bayly, R. C., Dagley, S. \& Gibson, D. T. (1966). The metabolism of cresol by species of Pseudomonas. Biochem J 101, 293-301.

Boyd, D. R., McMordie, R. A. S., Porter, P., Dalton, H., Jenkins, R. O. \& Howarth, O. W. (1987). Metabolism of bicyclic aza-arenes by Pseudomonas putida to yield vicinal cis-dihydrodiols and phenols. J Chem Soc Chem Commun 1987, 1722-1724.

Boyd, D. R., Blacker, J., Byrne, B., Dalton, H., Hand, M. V., Kelly, S. C., More O'Ferrall, R. A., Rao, S. N., Sharma, N. D. \& Sheldrake, G. N. (1994). Acid-catalyzed aromatisation of benzene cis-1,2dihydrodiols: a carbocation transition state poorly stabilised by resonance. J Chem Soc Chem Commun 1994, 313-314.

Colby, J., Stirling, D. I. \& Dalton, H. (1977). The soluble methane monooxygenase from Methylococcus capsulatus (Bath). Its ability to oxygenate $n$-alkanes, $n$-alkenes, ethers and alicyclic, aromatic and heterocyclic compounds. Biochem J 165, 395-402.

Dagley, S., Evans, W. C. \& Ribbons, D. W. (1960). New pathways in the oxidative metabolism of aromatic compounds by microorganisms. Nature 188, 560-566.

Dalton, H., Wilkins, P. C. \& Jiang, Y. (1993). Structure and mechanism of action of the hydroxylase of soluble methane monooxygenase. In Microbiol Growth on $C_{1}$ Compounds, pp. 65-80. Edited by J. C. Murrell and D. P. Kelly. Andover: Intercept.

Fredricks, K. M. (1967). Products of the oxidation of n-decane by Pseudomonas aeruginosa and Mycobacterium rhodochrous. Antonie Leeuwenhoek 33, 41-48.

Galli, E., Barbieri, P. \& Bestetti, G. (1992). Potential of pseudomonads in the degradation of methylbenzenes. In Pseudomonas Molecular Biology and Biotechnology, pp. 268-276. Edited by E. Galli, S. Silver \& B. Witholt. Washington, DC: American Society for Microbiology.

Gibson, D. T. \& Subramanian, V. (1984). Microbial degradation of aromatic hydrocarbons. In Microbial Degradation of Organic Compounds, pp. 181-252. Edited by D. T. Gibson. New York: Marcel Dekker.

Gibson, D. T., Koch, J. R., Schuld, C. L. \& Kallio, R. E. (1968). Oxidative degradation of aromatic hydrocarbons by microorganisms. II. Metabolism of halogenated aromatic hydrocarbons. Biochemistry 7, 3795-3802.

Gibson, D. T., Hensley, M., Yoshioka, H. \& Mabry, T. J. (1970). Formation of (+)-cis-2,3-dihydroxy-1-methylcyclohexa-4,6diene from toluene by Pseudomonas putida. Biochemistry 9, 1626-1630.

Gibson, D. T., Zylstra, G. J. \& Chauhan, S. (1990). Biotransformations catalysed by toluene dioxygenase from Pseudomonas putida F1. In Pseudomonas: Biotransformations, Pathogenesis and Evolving Biotechnology, pp. 121-132. Edited by S. Silver, A. M. Chakrabarty, B. Inglewski \& S. Kaplan. Washington, DC: American Society for Microbiology.

Grossebuter, W., Reiff, I. \& Rehm, H. J. (1979). Oxidation of ntetradecane by some Streptomyces spp. Eur J Appl Microbiol Biotechnol 8, 139-141.

Helmbrook, D. C. \& Sligar, S. G. (1981). Multiple mechanisms of cytochrome P450-catalysed substrate hydroxylations. Biochem Biophys Res Commun 99, 530-535.

Higson, F. K. \& Focht, D. D. (1992). Degradation of 2methylbenzoic acid by Pseudomonas cepacia MB2. Appl Environ Microbiol 58, 194-200.

Jenkins, R. O., Stephens, G. M. \& Dalton, H. (1987). Evidence for more than one form of toluene cis-glycol dehydrogenase from
Pseudomonas putida NCIB 11767. FEMS Microbiol Lett 44, 209-214.

Klein, D. A. \& Henning, F. A. (1969). Role of alcoholic intermediates in formation of isomeric ketones from $\mathrm{n}$ hexadecane by a soil Arthrobacter. Appl Microbiol 17, 676-681.

Kok, M., Shaw, J. P. \& Harayama, S. (1992). Comparison of two hydrocarbon monooxygenases of Pseudomonas putida. In Pseudomonas Molecular Biology and Biotechnology, pp. 214-222. Edited by E. Galli, S. Silver \& B. Witholt. Washington, DC: American Society for Microbiology.

Kukor, J. J. \& Olsen, R. H. (1990). Molecular cloning, characterization, and regulation of a Pseudomonas pickettii PKO1 gene encoding phenol hydroxylase and expression of the gene in Pseudomonas aeruginosa PAO1c. J Bacteriol 172, $4624-4630$.

Leadbetter, E. R. \& Foster, J. W. (1960). Bacterial oxidation of gaseous alkanes. Arch Mikrobiol 35, 92-104.

Mahajan, M. C., Phale, P. S. \& Vaidyanathan, C. S. (1994). Evidence for the involvement of multiple pathways in the biodegradation of 1- and 2-methylnaphthalene by Pseudomonas putida CSV86. Arch Microbiol 161, 425-433.

May, S. W. \& Abbott, B. J. (1972). Enzymatic epoxidation I. Alkene epoxidation by the $\omega$-hydroxylation system of Pseudomonas oleovorans. Biochem Biophys Res Commun 48, 1230-1234.

O'Donnell, K. J. \& Williams, P. A. (1991). Duplication of both $x y l$ catabolic operons on TOL plasmid pWW15. J Gen Microbiol 137, 2831-2838.

Schraa, G., Bethe, B. M., van Neerven, A. R. W., van den Tweel, W. J. J., van der Wende, E. \& Zehnder, A. J. B. (1987). Degradation of 1,2-dimethylbenzene by Corynebacterium strain C125. Antonie Leeuwenhoek 53, 159-170.

Shields, M. S., Montgomery, S. O., Chapman, P. J., Cuskey, S. M. \& Pritchard, P. H. (1989). Novel pathway of toluene catabolism in the trichloroethylene degrading bacterium G4. Appl Environ Microbiol 55, 1624-1629.

Stephens, G. M. \& Dalton, H. (1986). The role of the terminal and subterminal oxidation pathways in propane metabolism by bacteria. J Gen Microbiol 132, 2453-2462.

Stephens, G. M. \& Dalton, H. (1987). The effect of lipophilic weak acids on the segregational stability of TOL plasmids in Pseudomonas putida. J Gen Microbiol 133, 1891-1899.

Wackett, L. P., Kwart, L. D. \& Gibson, D. T. (1988). Benzylic monooxygenation catalyzed by toluene dioxygenase from Pseudomonas putida. Biochemistry 1988, 1360-1367.

Wende, P., Pfleger, K. \& Bernhardt, F.-H. (1982). Dioxygen activation by putidamonooxin: substrate-modulated reaction of activation oxygen. Biochem Biophys Res Commun 104, 527-532.

Williams, P. A. \& Murray, K. (1974). Metabolism of benzoate and the methylbenzoates by Pseudomonas putida (arvilla) mt-2: evidence for the existence of a TOL plasmid. J Bacteriol 120, 416-423.

Yen, K.-M., Karl, M. R., Blatt, L. M., Simon, M. J., Winter, R. B., Fausset, P. R., Lu, H. S., Harcourt, A. A. \& Chen, K. K. (1991). Cloning and characterization of a Pseudomonas mendocina KR1 gene cluster encoding toluene-4-monooxygenase. J Bacteriol 173, $5315-5327$.

Received 29 January 1997; accepted 20 February 1997. 